\title{
Factors Affecting the Decontamination Process in Hospitals in Saudi Arabia
}

This article was published in the following Dove Press journal:

Risk Management and Healthcare Policy

\author{
Ebaa M Felemban \\ Hanan AM Youssef \\ Abdulellah Al Thobaity (D) \\ Nursing Department, Applied Medical \\ Sciences, Taif University, Taif, Saudi \\ Arabia
}

Introduction: Hospitals face various types of disasters that require either decontamination or disinfection interventions. These contaminants can be chemical, biological, radioactive, or infectious, such as COVID-19. Further, there are few studies in the literature on factors affecting decontamination in hospitals in Saudi Arabia.

Methods: Approximately 157 healthcare providers (doctors, nurses, and other specialists) participated in this study. Principle component analysis was used to explore three factors in Saudi Arabia that affect the ability of healthcare providers to decontaminate appropriately.

Results: Three factors were extracted: (1) having adequate skills to perform decontamination, (2) being adequately prepared for decontamination before a disaster occurs, and (3) organizational barriers to decontamination. There was a positive correlation between the skills and preparedness and a negative correlation between barriers and both skills and preparedness.

Discussion: It is essential to prepare for decontamination during disasters more effectively and to ensure that all healthcare providers have the requisite skills. Moreover, barriers to decontamination must be investigated thoroughly to improve implementation.

Keywords: risk, disaster, COVID-19, healthcare providers

\section{Introduction}

Hospitals face disasters that can involve different contaminants, including biological, chemical, radiological, or infectious, such as COVID-19. ${ }^{1}$ This type of disaster requires specific plans and preparedness since they run a high risk of harming hospitals, patients, and healthcare providers from the contaminating substances., These contaminants can be in different physical forms (solid, liquid, or gas) and include bodily fluids such as saliva or blood. ${ }^{4,5}$ Decontamination strategies are some of the most important domains of planning and preparation for this type of disaster to remove the harmful substances and disinfect the patients or equipment. ${ }^{6,7}$

During an emergency situation or disaster, a quick response to cleaning and disinfecting patients ensures the safety of current hospital patients and healthcare providers by significantly reducing secondary contamination. ${ }^{1,8,9}$ However, some infected or contaminated patients arrive at the hospital in need of urgent medical intervention; hence, the timing of decontamination is crucial. The types and methods of decontamination also depend on the type of contaminant. The literature reports five important types of decontamination. First, dry decontamination is very useful for patients exposed to biological or radiation risks. This can be performed by removing the patient's clothes and giving them a full bath, washing their hands

Correspondence: Abdulellah Al Thobaity Nursing Department, Applied Medical Sciences, Taif University, Taif, Saudi Arabia Email a.thobaity@tu.edu.sa
Risk Management and Healthcare Policy 2021:14 357-363

DovePres: in $\square$ 
and face, then placing them in clean clothes before entering the hospital. The second type is eye decontamination, which only requires clean water for all situations (biological, chemical, radiation, or bodily fluids). The third type addresses patients who have been contaminated on external parts of the body, which can be removed using clean water and antiseptic soap. The fourth type of decontamination is used when the patient has ingested or inhaled naturalizing substances. Finally, the fifth type is mass decontamination of a large number of victims from a chemical disaster, where water is used prior to removing their clothes. ${ }^{1,5,10,11}$

The literature on decontamination for healthcare providers indicates that ensuring the availability of guidelines in addition to education and training regarding improving decontamination practice are recommended. ${ }^{12,13}$ Moreover, the literature available in Saudi Arabia suggests that health care providers already possess adequate knowledge regarding decontamination in terms of identifying suitable personal protective equipment (PPE). However, the participants lacked familiarity with decontamination procedures in their hospitals. ${ }^{14,15}$ Other issues related to decontaminating mass casualties in hospitals in Saudi Arabia are that the system is not fully developed and some hospitals do not have a designated building for decontamination (although this can be mitigated through the use of decontamination tents in front of emergency departments). ${ }^{16}$ Accordingly, it is essential to develop a more detailed plan by identifying the decontamination team, developing a protocol, and training the healthcare providers to be prepared. ${ }^{17}$ Several studies in Saudi Arabia have emphasized the importance of ensuring all hospitals are prepared for decontamination. This would include identifying the decontamination needs of each hospital, ensuring the availability of PPE, identifying factors for maintaining staff performance and privacy, determining a process for triaging the contaminated patients, planning for self-decontamination, and identifying those victims that may need extra assistance. ${ }^{14,18-25}$

Despite some studies in the literature that focused on the core knowledge of healthcare providers, preparedness, and planning for decontamination during crises in Saudi Arabia, evidence remains lacking in several areas. A notable omission is the factors affecting decontamination in hospitals. ${ }^{18,20-22,24,45}$ Understanding such factors will support the development of plans for all types of disasters that require decontamination and ensure the strongest readiness of healthcare providers to respond effectively. This would be achieved through developing educational programs, creating policies, training staff on how to perform decontamination correctly, buying essential equipment and supplies, and performing drill exercises based on specific disasters. Therefore, the aim of this study is to explore the factors that can improve decontamination in Saudi Arabian hospitals.

\section{Methods}

This research utilized a cross-sectional survey of all healthcare providers in major hospitals in Taif City, Saudi Arabia (excluding military hospitals). The population of this study is composed of healthcare providers who work in a hospital. A non-random convenience sample was obtained: all healthcare providers were involved in this study except those who had less than one year of experience in the hospital or were not able to read and write in English (the main language of all hospitals in Saudi Arabia). The number of samples was chosen based on principle component analysis (PCA) requiring a sample size $>100{ }^{26}$

The authors created a scale for collecting data from healthcare providers, which was rigorously evaluated for validity and reliability using PCA. Items were considered redundant and deleted if their loading was $<0.40$ on the factor or not loaded to any factor if there was a crossloading for one item on two factors. The values of reliability are reported in the results section. In terms of data collection, ethical approval was received from the IRB at the research department at the Directorate of Health Affairs in Taif (IRB registration number HAP-02-T-067 with approval number 434 from 06/09/2020). This approval was required by the selected hospitals despite the authors being from Taif University. The researchers collected data by arranging for the questionnaires to be distributed by the education and training coordinators of each hospital. An explanatory statement was provided for participants in addition to the survey. However, explicit consent was not sought from the participants-their completion and submission of the survey was considered sufficient. It should be stated that this study was conducted in accordance with the Declaration of Helsinki.

In total, 300 questionnaires were distributed in two hospitals. Based on the response rate, data were entered into Excel and then exported into SPSS (version 24) for analysis. ${ }^{27}$ Data were screened for missing data and outliers and then tested for normality by calculating the skewness and kurtosis of each item, which is required for PCA. Redundant items were deleted on the cross-loading of 
items with differences between primary and secondary loadings of $>0.30$ or if an item had no loading or a weak loading $(<0.40)$ on an associated factor. ${ }^{26}$

\section{Findings}

In this study, 157 healthcare providers participated by responding to the questions and returning the questionnaires, representing a total response rate of $52.33 \%$. The majority of the respondents were female $(>80 \%)$, with only 22 male participants. Four participants preferred not to disclose their gender. The age of the participants was classified into four groups, with approximately $50 \%$ being in the 30-39 year age group and 35\% in the 20-29 year age group. Accordingly, $>85 \%$ of the participants were $<40$ years old. The majority of the participants were nurses (78\%), with physicians only accounting for $10 \%$. More details of this analysis are presented in Table 1

Prior to PCA, data screening was normal and appropriate. The findings of PCA with varimax indicated that four items had to be removed as they cross-loaded with other factors. From the remaining 17 items, 3 factors were extracted as the following 6 items were loaded in the first factor with engine values of 7.92 and variance of 46.62 . The loading of the items ranged from 0.84 to 0.52 . All items reflected the confidence and ability of the healthcare providers, labelling their skills as performing decontamination. The descriptive finding for this factor was $\mathrm{M}=3.60, \mathrm{SD}=0.74$, and Cronbach's $\alpha$ value of 0.89 . The second factor contained seven items with Eigenvalues of 1.39 and a variance value of $8.20 \%$. The seven items loaded on this factor ranged from 0.72 to 0.55 . All items were related to participant preparedness for decontamination; therefore, this

Table I Demographics

\begin{tabular}{|l|l|l|l|}
\hline Groups & Subgroups & Frequency & Percent \\
\hline Gender & Female & 131 & 82.45 \\
& Male & 22 & $13.8 \%$ \\
& I prefer not to say & 4 & $2.5 \%$ \\
& Total & 157 & $98.7 \%$ \\
\hline \multirow{2}{*}{ Age } & $20-29$ & 58 & $36.5 \%$ \\
& $30-39$ & 78 & $49.1 \%$ \\
& $40-49$ & 18 & $11.3 \%$ \\
& $50-65$ & 3 & $1.9 \%$ \\
& Total & 157 & $98.7 \%$ \\
\hline \multirow{2}{*}{ Job } & Physician & 16 & $10.1 \%$ \\
& Nurse & 124 & $78.0 \%$ \\
& Others & 17 & $10.7 \%$ \\
& Total & 157 & $98.7 \%$ \\
\hline
\end{tabular}

factor was labeled as decontaminant preparedness, with $\mathrm{M}=$ $3.51, \mathrm{Sd}=0.74$, and a Cronbach's $\alpha$ value of 0.86 . The third factor comprised 7.94 from the total of variance with an eigenvalue of 1.35 and loading of 0.73 to 0.67 . Four items were loaded on this factor, which outlined the barriers to the decontamination process in hospitals (as perceived by the healthcare providers). This factor was labeled as barriers for decontamination with $\mathrm{M}=2.68, \mathrm{Sd}=0.81$, and Cronbach's $\alpha$ value of 0.77 . Table 2 shows the findings of PCA and loading of the extracted factors.

There was a strong positive correlation between skills and preparedness $(\mathrm{R}=0.70)$-increasing skills demonstrably enhances preparedness. The correlation between the skills and barrier factor was $\mathrm{R}=-0.55$, indicating how barriers decrease as healthcare providers' skills increase. Similarly, the correlation between preparedness and barriers was $\mathrm{R}=-$ 0.56 , which indicates that increased preparedness decreases barriers to contamination implementation. Table 3 shows the correlations between the factors.

\section{Discussion}

The findings of PCA indicate three factors were extracted from the data skills, preparedness and barriers to decontamination (as perceived by the healthcare providers). The majority of participants were female healthcare providers, which was expected as they dominate the nursing profession. The data of this study were validated through PCA where redundant items were removed, no loadings or cross-loadings were deleted, and all validated items with an accepted loading to a related factor remained. In terms of reliability, all constructed factors were reliable and valid in this study, as approved by the statistics. To ensure that healthcare providers are ready for any type of crisis (including pandemics such as COVID-19), increased effort must be made by hospital managers, disaster coordinators, decision makers, researchers, and educators to ensure that responses (including decontamination) are effective. $^{28-30}$

The first factor identified in this study was related to the core competencies of healthcare providers and their ability to decontaminate infected or contaminated victims with confidence. Decision makers internationally have recognized that ensuring all healthcare providers have the necessary skills is a major concern. Therefore, identifying areas for improvement is necessary in addition to helping healthcare providers understand the correct use and appropriateness of PPE. ${ }^{30}$ There are five types of decontamination: chemical, radiation, and biological decontamination, decontamination triage, and decontaminating hazardous substances. However, some important 
Table 2 Principles Component Analysis (PCA) Findings

\begin{tabular}{|c|c|c|c|c|c|}
\hline \multirow[t]{2}{*}{ Items } & \multicolumn{3}{|c|}{ Factors } & \multicolumn{2}{|c|}{ Descriptive } \\
\hline & $\mathbf{I}$ & 2 & 3 & $* \mathbf{M}$ & $* \mathbf{S D}$ \\
\hline I am confident enough to perform chemical decontamination. & 0.84 & 0.25 & -0.18 & 3.49 & 0.97 \\
\hline I am confident enough to perform radiation decontamination. & 0.82 & 0.14 & -0.08 & 3.17 & 1.08 \\
\hline I am confident enough to perform biological decontamination. & 0.80 & 0.28 & -0.22 & 3.55 & 0.96 \\
\hline I am able to do decontamination triage. & 0.73 & 0.39 & -0.19 & 3.60 & 0.89 \\
\hline I am able to implement the basic level of decontamination in my hospital. & 0.63 & 0.27 & -0.35 & 3.91 & 0.77 \\
\hline I am familiar with the hazardous substances in the hospital. & 0.52 & 0.35 & -0.25 & 3.92 & 0.81 \\
\hline Our hospital has a team responsible for decontamination tasks. & 0.35 & 0.72 & -0.26 & 3.55 & 1.01 \\
\hline The roles, responsibilities, and accountabilities related to decontamination are clear in our hospital. & 0.29 & 0.71 & -0.40 & 3.46 & 0.98 \\
\hline I received the training programs regarding decontamination in the workplace. & 0.24 & 0.70 & -0.18 & 3.69 & 1.02 \\
\hline $\begin{array}{l}\text { The set-up procedures for decontamination can be established outside my hospital during emergencies, } \\
\text { which involve hot, cold, and warm zones. }\end{array}$ & 0.23 & 0.70 & 0.06 & 3.32 & 0.86 \\
\hline Our hospital has an adequate number of decontamination experts. & 0.16 & 0.65 & -0.31 & 3.40 & 1.01 \\
\hline My organization supports the importance of developing a decontamination system. & 0.24 & 0.64 & -0.34 & 3.65 & 1.02 \\
\hline I learned the required decontamination core competencies from my undergraduate curriculum. & 0.13 & 0.55 & -0.04 & 3.49 & 1.09 \\
\hline $\begin{array}{l}\text { The hospital does not have sufficient tools and checklists to support me to implement the } \\
\text { decontamination strategies. }\end{array}$ & -0.23 & 0.01 & 0.73 & 2.89 & I.II \\
\hline The hospital management is not very interested in developing a decontamination system. & -0.32 & -0.29 & 0.72 & 2.42 & 1.05 \\
\hline The training opportunities I received on decontamination were ineffective. & -0.01 & -0.21 & 0.68 & 2.69 & 1.02 \\
\hline We lack standardized protocols at the hospital level in Saudi Arabia. & -0.33 & -0.25 & 0.67 & 2.71 & 1.06 \\
\hline Eigenvalue & 7.92 & 1.39 & 1.35 & - & - \\
\hline Variations \% & 46.62 & 8.20 & 7.94 & - & - \\
\hline
\end{tabular}

Note: The bold numbers are the loading weights $>0.40$.

Abbreviations: *M, mean; ${ }^{* S D}$, Standard deviation.

scales in Saudi Arabia have been developed to test nurses' core competencies in a disaster, which might be useful for decision makers to identify further training needs for healthcare providers.

Second, the concept of preparedness focuses on taking actions to ensure hospital and healthcare providers are ready for disasters. ${ }^{5,31}$ When there are mass casualties in emergency departments (such as when hospitals were overloaded by COVID-19 cases in many countries), decontamination through disinfection or cleaning becomes one of the most critical issues. ${ }^{32,33}$ To be well prepared, this study suggests building a decontamination team, which could involve incident commanders, logistics managers, medical directors, nursing directors, decontamination triage leaders, zone managers, and security. Further, identifying the roles and responsibilities of each member would prevent chaos by ensuring perfect performance and an effective response when cases arrive at the hospital..$^{5,8,17,28,31}$ Another issue related to the preparedness factor identified in this study was the notion that all hospitals must plan and implement more effective decontamination training. As summarized in the literature, some important topics in this training might include the following: removing the different types of harmful substance that might put patients and staff at risk of contamination; identifying the possible risks and harm from each substance or case; understanding the team and each person's role; knowing the supplies and equipment required for each decontamination method; and the ability 
Table 3 Correlations Between Factors

\begin{tabular}{|l|l|l|l|l|}
\hline \multicolumn{2}{|l|}{ Correlations } & Skills & Preparedness & Barriers \\
\hline Skills & Pearson correlation & 1 & $0.70^{* *}$ & $-0.55^{* *}$ \\
& Sig. (2-tailed) & 159 & 0.01 & 0.01 \\
& $\mathrm{~N}$ & 158 & 158 \\
\hline Preparedness & Pearson correlation & $0.654^{* *}$ & 1 & $-0.562^{* *}$ \\
& Sig. (2-tailed) & 0.01 & 158 & 0.01 \\
& $\mathrm{~N}$ & 158 & $-0.562^{* *}$ & 158 \\
\hline \multirow{2}{*}{ Barriers } & Pearson correlation & $-0.55 I^{* *}$ & 0.01 & 1 \\
& Sig. (2-tailed) & 0.01 & 158 & 158 \\
\hline
\end{tabular}

Note: ${ }^{* *}$ Correlation is significant at the 0.01 level (2-tailed).

to activate the plan for decontamination in addition to triaging contaminated patients when necessary. $5,8,16,19,21,28,30,31$

Finally, healthcare providers also acknowledged they faced barriers to their practice, the most important being insufficient tools and checklists. This issue has been reported by nurses in previous studies in Saudi Arabia. ${ }^{21,24,32}$ Healthcare providers in Saudi Arabia were confused about the types of PPE to use during the response to COVID-19. The availability of protocols and other specific tools would eliminate such confusion. ${ }^{34}$ Insufficient standardization of protocols and disaster planning has also been identified as a significant barrier. ${ }^{21,30,35-38}$ The ministry of health in Saudi Arabia highly recommends working with other health authorities to standardize the protocols and plans for all types of disasters. Lack of effective training is another issue that challenges healthcare providers in decontamination. As reported in the international literature, effective programs for Saudi hospitals must involve training for healthcare providers to enable them to perform the following: recognize hazardous substances, perform basic decontamination, implement advanced control of infection and contamination, use PPE effectively, and assess and treat infected patients effectively. ${ }^{19,30,31,39}$ All of the aforementioned measures will enhance preparedness and decrease the challenges faced by healthcare providers during decontamination procedures. Moreover, the strong relationship between skills and preparedness and the negative relationship between skills and barriers were identified in this study.

\section{Limitations}

This study investigated factors that affect the decontamination process in hospitals from the perspective of healthcare provided achieved through a survey conducted in two hospitals with a sample size of 157 . However, further efforts are required to contribute to understanding the factors in more detail.

\section{Conclusion}

Decontamination procedures for controlling contamination and infection in any disaster are paramount to the safety of hospitals, patients, and healthcare providers. However, healthcare providers perceive there are certain impediments that might affect the level of decontamination in their work environment, including a lack of skills, inadequate training, and insufficient preparedness. Improving these conditions to overcome the barriers will necessitate significant effort from decision makers, hospitals managers, disasters planners, researchers, and educators. This would ensure healthcare providers could maintain a high level of readiness to work without any difficulties during decontamination procedures.

\section{Acknowledgments}

We deeply thank the Deanship of Research at Taif University for their endless support and funding for this research (Project No: 1-441-67).

\section{Disclosure}

The authors report no conflicts of interest for this work.

\section{References}

1. Mortelmans LJM, Gaakeer MI, Dieltiens G, Anseeuw K, Sabbe MB. Are Dutch hospitals prepared for chemical, biological, or radionuclear incidents? A survey study. Prehosp Disaster Med. 2017;32 (5):483-491. doi:10.1017/S1049023X17006513

2. Grundmann O. The current state of bioterrorist attack surveillance and preparedness in the US. Risk Manag Healthc Policy. 2014;7:177-187.

3. Fornwalt L, Riddell B. Implementation of innovative pulsed xenon ultraviolet (PX-UV) environmental cleaning in an acute care hospital. Risk Manag Healthc Policy. 2014;7:25-28.

4. Baxter C, Gaskin S, Logan M, Pisaniello D. The mass decontamination paradigm: response relating to gas phase exposures and skin decontamination. Skin Decontamination. 2020;141-162.

5. Powers R, Daily E. International disaster nursing. Cambridge University Press. 2010. 
6. Levitin HW, Siegelson HJ, Dickinson S, et al. Decontamination of mass casualties: re-evaluating existing dogma. Prehosp Disaster Med. 2003;18(3):200-207.

7. Song X, Vossebein L, Zille A. Efficacy of disinfectant-impregnated wipes used for surface disinfection in hospitals: a review. Antimicrob Resist Infect Control. 2019;8:139.

8. Cox B. Hospital decontamination: what nurses need to know. Nursing Clinics. 2016;51(4):663-674.

9. Domres BD, Rashid A, Grundgeiger J, et al. European survey on decontamination in mass casualty incidents. Am J Disaster Med. 2009;4(3):147-152.

10. Titus E, Lemmer G, Slagley J, Eninger R. A review of CBRN topics related to military and civilian patient exposure and decontamination. Am J Disaster Med. 2019;14(2):137-149.

11. Calfee MW, Tufts J, Meyer K, et al. Evaluation of standardized sample collection, packaging, and decontamination procedures to assess cross-contamination potential during Bacillus anthracis incident response operations. J Occup Environ Hyg. 2016;13(12):980-992.

12. Sahiledengle B. Decontamination of patient equipment: nurses' selfreported decontamination practice in hospitals of southeast Ethiopia. BMC Res Notes. 2019;12:392. doi:10.1186/s13104-019-4427-5

13. Sahiledengle B. Instrument processing knowledge and practice amongst healthcare workers in Addis Ababa, Ethiopia. Int $J$ of Infection Cont. 2018;14:2.

14. Sultan MAS, Løwe Sørensen J, Carlström E, Mortelmans L, Khorram-Manesh A. Emergency healthcare providers' perceptions of preparedness and willingness to work during disasters and public health emergencies. Healthcare (Basel). 2020;8:4.

15. AlHarastani HAM, Alawad YI, Devi B, et al. Emergency and disaster preparedness at a tertiary medical city. Disaster Med Public Health Prep. 2020;1-11.

16. Qureshi MN, AlRajhi A. Challenge of COVID-19 crisis managed by emergency department of a big tertiary centre in Saudi Arabia. Int J Pediatr Adolesc Med. 2020;7(3):147-152.

17. Al Thobaity A, Alamri S, Plummer V, Williams B. Exploring the necessary disaster plan components in Saudi Arabian hospitals. Int J Disaster Risk Red. 2019;41:101316.

18. Alshehri A, Al Moteri Modi O, Plummer V, Endacott R, Al Thobaity A. Using logic model and key performance indicators to construct a field hospital for coronavirus pandemic cases. $B M C$ Emerg Med. 2020.

19. Al Thobaity A, Plummer V, Williams B. What are the most common domains of the core competencies of disaster nursing? A scoping review. Int Emerg Nurs. 2017;31:64-71.

20. Al Thobaity A, Plummer V, Innes K, Copnell B. Perceptions of knowledge of disaster management among military and civilian nurses in Saudi Arabia. Australas Emerg Nurs J. 2015;18 (3):156-164

21. Al Thobaity A, Williams B, Plummer V. A new scale for disaster nursing core competencies: development and psychometric testing. Australas Emerg Nurs J. 2016;19(1):11-19.

22. Alzahrani F, Kyratsis Y. Emergency nurse disaster preparedness during mass gatherings: a cross-sectional survey of emergency nurses' perceptions in hospitals in Mecca, Saudi Arabia. BMJ Open. 2017;7 (4):e013563.
23. Baker OG, Alamri AA, Aboshaiqah AE. A descriptive study to analyse the disaster preparedness among Saudi nurses through self-regulation survey. J Nurs Manag. 2019;27(7):1479-1484.

24. Al Harthi M, Al Thobaity A, Al Ahmari W, Almalki M. Challenges for nurses in disaster management: a scoping review. Risk Manag Healthc Pol. 2020;13:2627-2634.

25. Selam MN, Bayisa R, Ababu A, et al. Increased production of alcohol-based hand rub solution in response to COVID-19 and fire hazard potential: preparedness of public hospitals in Addis Ababa, Ethiopia. Risk Manag Healthc Pol. 2020;13:2507-2513.

26. Williams B, Onsman A, Brown T. Exploratory factor analysis: a five-step guide for novices. Australas Jl of Paramedicine. 2010;8:3.

27. IBM Corp. Released 2016. IBM SPSS Statistics for Macintosh, Version 24.0. Armonk, NY: IBM Corp.

28. Abbasabadi Arab M, Khankeh HR, Mosadeghrad AM, Farrokhi M. Developing a hospital disaster risk management evaluation model. Risk Manag Healthc Policy. 2019;12:287-296.

29. Park D, Ha J. Comparison of COVID-19 and MERS risk communication in Korea: a case study of tv public service advertisements. Risk Manag Healthc Policy. 2020;13:2469-2482.

30. Schultz CH, Koenig KL, Whiteside M, Murray R.Development of national standardized all-hazard disaster core competencies for acute care physicians, nurses, and EMS professionals. Ann Emerg Med. 2012;59(3):196-208.

31. Powers R. Decontamination. In: Daily E, Powers R, editors. International Disaster Nursing. Cambridge: Cambridge University Press; 2010:265-288.

32. Al Thobaity A, Alshammari F. Nurses on the frontline against the COVID-19 pandemic: an integrative review. Dubai Med J. 1-6.

33. Orsini E, Mireles-Cabodevila E, Ashton R, Khouli H, Chaisson N. How we do it: lessons on outbreak preparedness from the Cleveland clinic. Chest. 2020.

34. AlAssaf W. Challenges in pandemic disaster preparedness: experience of a Saudi academic medical center. Disaster Med Public Health Prep. 2020;1-5.

35. Karimkhani C, Amir M, Dellavalle RP, Ipaktchi K. Current concepts for oil decontamination of crush injuries: a review. Patient Saf Surg. 2014;8:22.

36. Reidy M, Ryan F, Hogan D, Lacey S, Buckley C. Preparedness of hospitals in the Republic of Ireland for an influenza pandemic: an infection control perspective. BMC Public Health. 2015;15:847.

37. Ingram AE, Hertelendy AJ, Molloy MS, Ciottone GR. State preparedness for crisis standards of care in the United States: implications for emergency management. Prehosp Disaster Med. 2020;1-3.

38. Dell'Era S, Hugli O, Dami F. Hospital disaster preparedness in Switzerland over a decade: a national survey. Disaster Med Public Health Prep. 2019;13(3):433-439.

39. Sarin RR, Cattamanchi S, Alqahtani A, Aljohani M, Keim M, Ciottone GR. Disaster education: a survey study to analyze disaster medicine training in emergency medicine residency programs in the United States. Prehosp Disaster Med. 2017;32(4):368-373. 


\section{Publish your work in this journal}

Risk Management and Healthcare Policy is an international, peerreviewed, open access journal focusing on all aspects of public health, policy, and preventative measures to promote good health and improve morbidity and mortality in the population. The journa welcomes submitted papers covering original research, basic science, clinical \& epidemiological studies, reviews and evaluations, guidelines, expert opinion and commentary, case reports and extended reports. The manuscript management system is completely online and includes a very quick and fair peer-review system, which is all easy to use. Visit http://www.dovepress.com/testimonials.php to read real quotes from published authors.

Submit your manuscript here: https://www.dovepress.com/risk-management-and-healthcare-policy-journal 